\title{
Preparation of Chain-End Clickable Recombinant Protein and Its
}

\section{Bio-Orthogonal Modification}

Lin Wang ${ }^{1}$, Rui Jiang ${ }^{1,2}$, Lin Wang ${ }^{1,3}$, Yang Liu $^{1,4}$, Xue-Long Sun ${ }^{1^{*}}$

${ }^{1}$ Department of Chemistry, Chemical and Biomedical Engineering and Center for Gene Regulation in Health and Disease (GRHD), Cleveland State University, Cleveland, Ohio, U.S.A.

${ }^{2}$ College of Life and Health Sciences, Northeastern University, Shenyang, 110004, P. R. China.

${ }^{3}$ Department of Medicinal Chemistry, China Medical University, Shenyang, China.

${ }^{4}$ Key Laboratory of Structure-Based Drugs Design \& Discovery of Ministry of Education, School of Pharmaceutical Engineering, Shenyang Pharmaceutical University, Shenyang, P. R. China

To be submitted to BIOORGANIC CHEMISTRY

*Correspondence: Xue-Long Sun, E-mail: x.sun55@csuohio.edu (X.-L. Sun) 


\section{Abstract:}

Introducing unique functional group into protein is an attractive approach for site-selective protein modification applications. In this report, we systemically investigated four siteselective strategies to introduce azide functionality into recombinant thrombomodulin $\left(\mathrm{TM}_{456}\right)$, via direct recombinant expression with unnatural amino acid, chemical, and enzymatic modification for its bio-orthogonal modification application. First, a straightforward recombinant method to express $\mathrm{TM}_{456}$ with azide functionality near $\mathrm{C}$ terminus by replacing methionine with azidohomoanlanine from methionine auxotroph $E$. coli cell was investigated. Next, a sortase-mediated ligation (SML) method to incorporate azide functionality into the $\mathrm{C}$-terminus of recombinant $\mathrm{TM}_{456}$ was demonstrated. The third is to add azide functionality to the $\mathrm{N}$-terminal amine of recombinant $\mathrm{TM}_{456}$ via amidation chemistry, and the fourth is tyrosine selective three-component Mannich reaction to introduce azide functionality to recombinant $\mathrm{TM}_{456}$. Overall, SML of recombinant protein affords the highest overall yield for incorporating azide functionality into the C-terminus recombinant $\mathrm{TM}_{456}$ since the key protein expression step uses natural amino acids. Also, single site modification facilitates the highest $\mathrm{TM}_{456}$ activity.

Keywords: azide; bio-orthogonal modification; copper-free click chemistry; sortase A; thrombomodulin 


\section{Introduction}

Modification of protein is a very important and necessary approach for studying protein structure and function, and the mechanisms of biological pathways that the protein is involved in. Furthermore, modification can expand protein's functional capacity, especially for therapeutic proteins for enhanced pharmacodynamic and pharmacokinetic properties. The key point for protein modification is to carry out a well-defined site-selective chemistry without reducing the protein's original activity and in high efficiency for generating homogeneous product. Bio-orthogonal chemistry facilitates site-selective modification by targeting to a specific functionality and has shown great potential for protein modifications of different interests. Azide has been proved as a versatile bio-orthogonal chemical reporter, which is small and inert in physiological settings and used for modifying diverse classes of biomolecules [1-5]. In particular, azide-based ligation reactions have been extensively explored for highly selective and biocompatible modification of proteins for biomedical applications [6-8]. Nowadays, three major bio-orthogonal reactions utilizing azide functionality have been fully developed, including Staudinger ligation, click chemistry and copper-free click chemistry (CFCC), among which CFCC is the most convenient and efficient one. Therefore, efficiently introducing azide functionality into proteins has become the key step and versatile approach for site-selective protein modification for both research and applications.

Thrombomodulin (TM) is a membrane glycoprotein mainly expressed by vascular endothelial cells as a major anticoagulation component [9]. TM consists of 557 amino acids divided into five distinct domains: $N$-terminal lectin-like domain (designated as TMD1); six epidermal growth factor (EGF)-like domain (TMD2); Ser/Thr-rich domain (TMD3); transmembrane domain (TMD4); and cytoplasmic tail domain (TMD5). The different domains are responsible for different biological functions of TM [10]. In the past decades, 
various domains of TM have been cloned and expressed for TM structural and functional study [11]. Further, recombinant TMs of different domains show promising antithrombotic and anti-inflammatory activity in both rodents and primates models. A recombinant soluble TM has been approved for therapeutic application in Japan [12]. In addition, TM has been considered as an excellent candidate for biomedical engineering applications. Chaikof et al. reported a truncated TM construct with an azidohomoalanine near $C$-terminus for siteselective modification via Staudinger ligation [13] and oriented immobilization through click chemistry [2]. Besides, bioactive modification of pancreatic islets with the azide-containing TM via Staudinger ligation was investigated by the same group, showing the potential to reduce procoagulant and pro-inflammatory responses upon transplantation $[14,15]$. All these results demonstrated the feasibility of recombinant azide-TM for cell surface re-engineering and its promising application for cell transplantation. Recently, we proposed a recombinant TM-liposome conjugate as a bio-inspired antithrombotic agent, which mimics both the protein and lipid membrane structure of the native endothelial membrane TM [16]. Briefly, a TM of EGF-like 4-6 domains with an azidohomoalanine near $C$-terminus $\left(\mathrm{rTM}_{456}-\mathrm{N}_{3}\right)$ was expressed via replacing methionine with azidohomoanlanine from methionine auxotroph $E$. coli cell. Then, site-selective conjugation of the $\mathrm{rTM}_{456}-\mathrm{N}_{3}$ with liposome via Staudinger ligation and $\mathrm{CFCC}$ afforded recombinant $\mathrm{TM}_{456}$-liposome conjugates, both of which showed enhanced protein $\mathrm{C}$ activation activity facilitated by lipid membrane.

Introducing unnatural amino acids into recombinant TM provides versatile tool for siteselective chemistry for protein modification applications. However, the low level of protein expression with unnatural amino acid limits its practical applications. Therefore, to find a way to introduce azide into protein efficiently and site-selectively is highly needed for all promising researches and applications of proteins like $\mathrm{TM}_{456}$ described above. In this study, we systemically investigated recombinant, enzymatic and chemical strategies to introduce 
azide functionality into $\mathrm{TM}_{456}$ site-selectively at either $N$-terminus or $C$-terminus. First, we investigated recombinant expression of recombinant $\mathrm{TM}_{456}$ with azide functionality near $C$ terminus by replacing methionine with azidohomoanlanine from methionine auxotroph $E$. coli cell. Second, we expressed $\mathrm{TM}_{456}$ with sortase A recognizing motif LPETG for introducing azide functionality into the $C$-terminus via sortase-mediated ligation (SML). Third, a chemical method was used to add azide molecule to the $N$-terminal amine of $\mathrm{TM}_{456}$ via amidation chemistry. Finally, multiple azide functional groups were introduced into $\mathrm{TM}_{456}$ via a tyrosine-selective three-component Mannich reaction (Figure 1). The azido$\mathrm{TM}_{456}$ derivatives obtained in all four methods were confirmed for site-selective modification and immobilization of recombinant TM through CFCC successfully.

\section{Figure 1}

\section{Materials and methods}

\subsection{Materials and Reagents}

Unless stated otherwise, all reagents and chemicals were obtained from commercial sources and used without further purification. pET $39 \mathrm{~b}$ vector, $\mathrm{pET} 28 \mathrm{~b}$ vector, competent cells, and kanamycin sulfate were purchased from EMD Chemicals (Philadelphia, PA). The mouse monoclonal antibody specific to human TM and Goat Anti-Mouse IgG H\&L (FITC) were purchased from Abcam (Cambridge, MA). Human protein C, human thrombin and human antithrombin III were obtained from Haematologic Technologies Inc.. L-azidohomoalanine was from AnaSpec Inc. (Fremont, CA). Chromogenic thrombin substrate BIOPHEN-CS01 was obtained from Aniara. Anti-PEG monoclonal Ab E11 was purchased from Academia

Sinica (Taipei, Taiwan). 4N-BOC-Gly-Gly-OH and N-hydroxysuccinimide were purchased 
from Sigma-Aldrich (St.Louis, USA). DBCO-Cy5, Azido-PEG 3 -Amine, Azido-PEG 4 -NHS Ester and DBCO-PEG ${ }_{5000}-\mathrm{OMe}$ were purchased from Click-Chemistry Tools (Scottsdale, AZ).

\subsection{Expression of FLAG-TM3}

The gene encoding the EGF domain 4-6 of human thrombomodulin with a FLAG tag (FLAG-TM3) and a C-terminal LPETG motif was designed and synthesized (Genscript Inc.). In order to prevent oxidation and reduce proteolytic susceptibility, three residues of TM were mutated (M388L, R456G and H457Q) as described previously [17]. The FLAG-TM3 gene was then transferred into pET-28b vector containing a C-terminal His-tag between NcoI and XhoI sites to obtain the expression plasmid pET28b-FLAG-TM3.

The expression plasmid and pET28b-FLAG-TM3 was transformed into E. coli. B834 (DE3) cells for expression. The E. coli.B834 (DE3) cells containing the expression plasmids were incubated in LB medium with $35 \mathrm{mg} / \mathrm{L}$ kanamycin at $37{ }^{\circ} \mathrm{C}$ until an $\mathrm{OD}_{600}$ of 0.8 was reached, and then IPTG was added to a final concentration of $1 \mathrm{mM}$ to induce the overexpression of $\mathrm{TM}_{456}$ by incubation for $5 \mathrm{hrs}$ at $37{ }^{\circ} \mathrm{C}$. The bacteria were then centrifuged at $8,000 \mathrm{~g}$ for $5 \mathrm{~min}$, and the cell pellet was collected and stored in $-20{ }^{\circ} \mathrm{C}$.

\subsection{Expression of azido-FLAG-TM3}

For azido-FLAG-TM3 expression, the E. coli.B834 (DE3) cells were incubated at $37{ }^{\circ} \mathrm{C}$ in M9AA medium (1 L) supplemented with $1 \mathrm{mM} \mathrm{MgSO}_{4}, 0.1 \mathrm{mM} \mathrm{CaCl} 2,0.4 \%$ (wt/v) glucose, $1 \mathrm{mg} / \mathrm{L}$ thiamine chloride, $35 \mathrm{mg} / \mathrm{L}$ kanamycin and proteinogenic amino acids to the point that $\mathrm{OD}_{600}$ value around 0.8 . Methionine was then removed from the medium by centrifugation for $10 \mathrm{~min}$ at $4000 \mathrm{x} \mathrm{g}$ at room temperature, and the cell pellet was washed with M9AA medium. The pellet was resuspended in $1 \mathrm{~L}$ of the M9AA medium with all the 
components mentioned above except methionine and further incubated at $37^{\circ} \mathrm{C}$ for $1 \mathrm{~h}$ to starve the cells. L-azidohomoalanine was then added to the incubation medium to a final concentration of $80 \mathrm{mg} / \mathrm{L}$ and after incubation for another $1 \mathrm{~h}$, protein expression was induced by $1 \mathrm{mM}$ IPTG at $37^{\circ} \mathrm{C}$ for $5 \mathrm{~h}$. The cell culture was then centrifuged at $8,000 \mathrm{~g}$ for 5 min and the pellet was collected and kept in $-20{ }^{\circ} \mathrm{C}$ until use.

\subsection{Purification of TM3 and azido-TM3}

The cell pellet containing FLAG-TM3 and azido-FLAG-TM3 from above was resuspended in $40 \mathrm{~mL}$ of ice-cold lysis buffer $(20 \mathrm{mM}$ Tris and $150 \mathrm{mM} \mathrm{NaCl}, \mathrm{pH} 8.0)$ and lysed by sonication. The extract was then loaded into HisTrap FF column (GE Healthcare) charged with $\mathrm{Ni}^{2+}$ ions and eluted with buffer $(20 \mathrm{mM}$ Tris, $0.5 \mathrm{M} \mathrm{NaCl}$ and $250 \mathrm{mM}$ imidazole, $\mathrm{pH}$ 8.0). Pooled fractions containing recombinant $\mathrm{TM}_{456}$ were collected and then dialyzed against $20 \mathrm{mM}$ Tris and $150 \mathrm{mM} \mathrm{NaCl}, \mathrm{pH} 8.0$ to afford $\mathrm{TM}_{456}$ or azido-TM456 protein with LPETG motif. The FLAG tag of FLAG-TM3 or azido-FLAG-TM3 was further removed by cleavage of enterokinase, and the released TM3 or azido-TM3 was then purified by using HisTrap FF chromatography.

\subsection{Incorporation of azide to TM3 via sortase-mediated ligation}

TM3 $(10 \mu \mathrm{M})$ was mixed with SrtA $(5 \mu \mathrm{M})$ and diGly-PEG ${ }_{3}$-azide $(100 \mu \mathrm{M})$ in reaction buffer (20 mM Tris, $150 \mathrm{mM} \mathrm{NaCl}$ and $5 \mathrm{mM} \mathrm{CaCl}_{2}, \mathrm{pH}$ 8.0). The reaction mixture was then incubated at $37^{\circ} \mathrm{C}$ for $2 \mathrm{~h}$ to obtain the C-terminal modified TM3 (azido-TM3-S). Excess diGly-PEG 3 -azide was then removed by HiTrap desalting column (GE Healthcare). The obtained azido-TM3-S was further purified from the remaining reaction mixture by collecting the pass through fraction of HisTrap FF column. The total amount of pure azido-TM3-S was then measured after the purification. 


\subsection{Incorporation of azide to TM3 via amidation chemistry}

NHS-PEG 4 -azide was added to TM3 $(10 \mu \mathrm{M})$ in HEPEs buffer $\mathrm{pH} 7.4$ at a final concentration of $1 \mathrm{mM}$ and the reaction mixture was incubated at room temperature for $2 \mathrm{~h}$ and then quenched by adding $50 \mathrm{mM}$ Tris buffer for $5 \mathrm{~min}$ at room temperature. Then excess nonreacted $\mathrm{NHS}_{-} \mathrm{PEG}_{4}$-azide was removed by using a HiTrap Desalting column (GE healthcare) to afford azido-TM3-N.

\subsection{Incorporation of azide to TM3 via three-component Mannich reaction}

$200 \mu \mathrm{L}$ of 4 -Azidoanaline solution $(5 \mu \mathrm{mol}$ in $0.1 \mathrm{M}$ phosphate buffer, $\mathrm{pH} 6.5),, 200 \mu \mathrm{L}$ of formaldehyde solution ( $5 \mu \mathrm{mol}$ in $0.1 \mathrm{M}$ phosphate buffer, $\mathrm{pH} 6.5)$, and $200 \mu \mathrm{L}$ of TM3 solution (5 nmol in 0.1 M phosphate buffer, $\mathrm{pH}$ 6.5) were added to a microcentrifuge tube and mixed well. The reaction was performed at room temperature for $48 \mathrm{~h}$. The excess unreacted 4-azidoanaline and formaldehyde were then removed by HiTrap Desalting column to obtain azido-TM3-T.

\subsection{Fluorescent labeling of azido-TM456}

To a solution of azido- $\mathrm{TM}_{456}(10 \mu \mathrm{M}), \mathrm{DBCO}-\mathrm{Cy} 5$ was added to a final concentration of 20 $\mu \mathrm{M}$. The mixture was gently mixed at room temperature for $2 \mathrm{~h}$ in the dark. After the coupling reaction, the unreacted DBCO-Cy5 was removed by dialysis using centrifuge devices with a cutoff molecular weight of 10,000 Da. The successful ligations were then confirmed by SDS-PAGE and fluorescent images which was obtained by Typhoon 9410 fluorescence scanner $(E x / E m=633 / 670 \mathrm{~nm})$. The in-gel fluorescence intensities were calculated and compared by Image $\mathrm{J}$. 


\subsection{Protein C activation activity assay of $\mathrm{TM}_{456}$ derivatives}

The cofactor activities of $\mathrm{TM}_{456} \mathrm{~S}$ and azido- $\mathrm{TM}_{456} \mathrm{~S}$ were assessed by protein $\mathrm{C}$ activation assay as previously described with some modification [18]. $\mathrm{TM}_{456}$ derivatives obtained from above were added into assay buffer (20 mM Tris, $150 \mathrm{mM} \mathrm{NaCl}$ and $5 \mathrm{mM} \mathrm{CaCl}_{2}, \mathrm{pH} 8.0$ ) containing $200 \mathrm{nM}$ of human protein $\mathrm{C}$ (PC) to a final concentration of $50 \mathrm{nM}$, and the reaction volumes were adjusted to $100 \mu \mathrm{L}$. The $\mathrm{PC}$ activations were initiated with the addition of human $\alpha$-thrombin to a final concentration of $10 \mathrm{nM}$. After incubation for $1 \mathrm{~h}$ at $37^{\circ} \mathrm{C}$, the PC activation was terminated by addition of $30 \mu \mathrm{L}$ human antithrombin III (1 $\mathrm{mg} / \mathrm{mL})$ and $2 \mu \mathrm{L}$ heparin $(10 \mathrm{U} / \mathrm{mL})$ for $10 \mathrm{~min}$ at $37^{\circ} \mathrm{C}$. The activated $\mathrm{PC}$ was measured by incubation with chromogenic substrate H-D-Phe-Pip-Arg-pNa $(0.5 \mathrm{mM})$ to determine its enzymatic activity. The increases in UV absorbance at $405 \mathrm{~nm}$ were measured after $20 \mathrm{~min}$ incubation.

\subsection{Immobilization of azido-TM456 on DBCO-coated glass}

DBCO-coated glass was prepared by coating the aminosilanate glass slide with $\mathrm{DBCO}-\mathrm{PEG}_{4}-$ NHS ester (S2). For immobilization of azido-TM2 via CFCC, azido-TM2 was labeled by FITC via amine group on azido-TM2, and then $10 \mu \mathrm{M}$ of the FITC-labeled azido-TM2 was incubated with DBCO-coated glass slides $(1.5 \times 1.5 \mathrm{~cm})$ in reaction buffer $(20 \mathrm{mM}$ Tris- $\mathrm{HCl}$, $\mathrm{pH} 8.0,150 \mathrm{mM} \mathrm{NaCl}$ ) for overnight at room temperature. Next, the reaction buffer was removed from glass slide and the surface was washed with the PBS (pH 7.4) for three times to remove unreacted azido-TM2. The successful immobilization was confirmed upon examining the fluorescence image of FITC-labeled azido-TM2 $(\mathrm{Ex} / \mathrm{Em}=480 / 520 \mathrm{~nm})$.

For immobilization of azido-TM3 derivatives (azido-TM3-S, azido-TM3-N and azidoTM3-T) via CFCC, anti-TM antibody was used to detect the immobilized azido-TM3. Each 
azido-TM3 $(10 \mu \mathrm{M})$ in reaction buffer $(20 \mathrm{mM}$ Tris- $\mathrm{HCl}, \mathrm{pH} 8.0,150 \mathrm{mM} \mathrm{NaCl})$ was added on the DBCO-coated glass slides $(1.5 \times 1.5 \mathrm{~cm})$ and incubated for overnight at room temperature. Then the reaction buffer was removed from glass slide and the surface was washed with the PBS ( $\mathrm{pH}$ 7.4) for three times to remove unreacted azido-TM3 derivatives. Then, mouse anti-TM antibody was used as the first antibody to capture azido-TM3 derivatives, and followed by the secondary anti-body which is FITC-labeled anti-mouse antibody. Each antibody was incubated with the slide for $1 \mathrm{~h}$ at room temperature, and washed three times with PBS (pH 7.4). The successful immobilization was confirmed upon examining the fluorescence image of FITC-labeled anti-mouse antibody $(\mathrm{Ex} / \mathrm{Em}=480 / 520$ $\mathrm{nm})$.

\subsection{Protein $C$ activation activity assay of immobilized $T_{456}$ on DBCO-coated glass}

The protein $\mathrm{C}$ activation activities of immobilized $\mathrm{TM}_{456}$ (azido-TM2, azido-TM3-S, azidoTM3-N and azido-TM3-T) on glass slide were measured by protein $\mathrm{C}$ activation assay as previously described with some modification [18]. Assay buffer (20 mM Tris, $150 \mathrm{mM} \mathrm{NaCl}$ and $5 \mathrm{mM} \mathrm{CaCl}_{2}, \mathrm{pH} 8.0$ ) containing $200 \mathrm{nM}$ of human protein $\mathrm{C}(\mathrm{PC})$ and $10 \mathrm{nM}$ human $\alpha$ thrombin were added to the surface of DBCO-coated glass slide $(1.5 \times 1.5 \mathrm{~cm})$ with immobilized $\mathrm{TM}_{456}$. After $1 \mathrm{~h}$ incubation at $37^{\circ} \mathrm{C}$, the reaction was terminated by adding 30 $\mu \mathrm{L}$ of human antithrombin III $(1 \mathrm{mg} / \mathrm{mL})$ and $2 \mu \mathrm{L}$ of heparin $(10 \mathrm{U} / \mathrm{mL})$ and incubated for 10 min at $37^{\circ} \mathrm{C} .200 \mu \mathrm{L}$ of the assay buffer containing activated human protein $\mathrm{C}$ was transfer from the surface of DBCO-coated glass to microcentrifuge tube. The enzymatic activity of activated PC was measured by using chromogenic substrate H-D-Phe-Pip-Arg-pNa (0.5 $\mathrm{mM})$, and the UV absorbance at $405 \mathrm{~nm}$ was measured after 20 min incubation.

\section{Results}

\subsection{Expression and purification of recombinant TM3 and recombinant azido-TM3}


In order to express protein bearing unnatural amino acid, it requires the prokaryotic expression system with auxotrophic strain. However, due to the multiple disulfide bonds in $\mathrm{TM}_{456}$, it is challenging to express in E. coli because that the protein will either does not express at all or expressed as inclusion body. Previously, Chaikof et al [13] successfully expressed the soluble azido-TM456 in $E$. coli $\mathrm{B} 834$ cell which is a methionine auxotrophic strain by fusing $\mathrm{TM}_{456}$ to a DsbA tag in pET39b vector (TM1). Recently, we reported a recombinant $\mathrm{TM}_{456}$ with a LPETG tag at the $C$-terminus used for SML [19]. After expression in E. coli B834 cell, we obtained the fusion protein DsbA$\mathrm{TM}_{456} \mathrm{AL}$ (named DsbA-TM2 in the current study) as a final product by enzymatic removal of DsbA tag. This $\mathrm{TM}_{456}$ has the advantage to be modified by two distinguished functional groups through one-pot double modification via CFCC and enzymatic ligation (SML). Fusing with DsbA tag could enhance the correct folding and reduce the formation of inclusion body. However, we found that the expression yield of azido-DsbA-TM2 fusion protein is lower than native DsbA-TM2 expressed (Table 1). Further, a notable proportion of recombinant TM2 leaked into the culture medium, even at optimized expression temperature and time. Thus, only limited amount of TM2 was obtained. Moreover, DsbA tag had to be removed by thrombin cleavage to obtain the final TM2/azido-TM2, which adds extra purification steps. Therefore, we designed and expressed a new recombinant TM3 with FLAG fusion tag at the $N$-terminus and His-tag at the $C$-terminus, which can be expressed in cytoplasma to reduce the protein leaking. The designed gene fragment was inserted into pET28b (Novagen) plasmid and transformed into E. coli B834 cell for expression (Figure 2A and 2B). The expression yield of the native FLAG-TM3 is much higher than that of native DsbA-TM2, especially after tag removal, thus more native TM3 was obtained than native TM2. However, the expression yield of azido-TM3 was lower than azido-TM2 for unknown reasons (Table 1). There was no inclusion body observed by SDS-PAGE analysis of the 
precipitate of cell culture. Therefore, we postulated that FLAG-TM3 with higher expression yield by native $\mathrm{TM}_{456}$ expression can be used for the incorporation of azide via subsequent enzymatic and chemical modification.

Table 1

Figure 2

\subsection{Incorporation of azide into TM3 via enzymatic method}

First, we investigated an enzymatic method, sortase-mediated ligation (SML) to introduce azide group to $\mathrm{TM}_{456}$ (TM3) at the $C$-terminus. Sortase $\mathrm{A}$ is a transpeptidase used by Grampositive bacteria to anchor surface proteins to the cell wall through a condensation reaction between a $C$-terminal LPXTG motif and a $N$-terminal poly-(glycine) molecule [20]. The enzyme cleaves the LPXTG at the amide bond between the threonine and the glycine to form an acyl-enzyme intermediate. Subsequent nucleophilic attack by the amino group of the poly(glycine) to the intermediate results in the formation of an LPXT-G bond and the release of the enzyme. In recent years, several groups including us reported the protein modification by using SML, such as fluorescent labeling, glycosylation, PEGylation and lipidation [19,2124]. It has been reported that maximum reaction efficiency is generally reached by substrates with two glycines, the addition of any more glycines having no significant effect on the reaction rate [25]. Therefore, diglycine was chosen as the nucleophile in this study. By utilizing SML, azide group was introduced by a small molecule, diGly- $\mathrm{PEG}_{3}$-azide which has a good solubility in aqueous solution and high reaction yield. With this azide introduced, 
targeting functionality with larger group or poor solubility such as PEG molecule or lipid will be easily conjugated to the protein by CFCC next.

In this study, fusion $\mathrm{TM}_{456}$ and SrtA with a His-tag at the $C$-terminus were prepared for easy cleanup of the unreacted TM3 and SrtA after the SML reaction. As shown in Figure 3A, due to the cleavage of T/G in LPETG motif after the SML reaction, the product azido-TM3-S did not have the His-tag, while both the unreacted TM3 and SrtA still containing the His-tag at the $C$-terminus could be removed from the reaction mixture by nickel affinity column so as to afford the pure azido-TM3-S efficiently. As confirmed in SDS-PAGE, the azido-TM3-S without His-tag was obtained in the flow through fraction (Figure 3B. Lane 2), and both the unreacted TM3 and SrtA were in the eluate fraction (Figure 3B, Lane 3). By comparing the amount of TM3 before SML and the pure azido-TM3-S obtained after SML, the ligation yield was around 60\%. Overall, it afforded twice amount of azido-TM3-S (8.1 mg per one liter of cell culture) than direct expressed azido-TM2 (4.1 mg per one liter of cell culture) (Table 1). Next, DBCO-Cy5 was used to selectively react with azide group through CFCC confirming the successful incorporation of azide group into TM3 and its accessibility as well. As shown in figure 4A, the azido-TM3-S could be detected in fluorescent scanner as a bright red band after the fluorescent labeling. This result indicated the successful incorporation of azide functionality into a native $\mathrm{TM}_{456}$ through SML, which could provide large amount for $\mathrm{TM}_{456}$ modification applications.

\section{Figure 3}

\subsection{Incorporation of azide into TM3 via chemical methods}


Chemical modification of a native amino acid or terminals in the protein has been the main choice in the past [26]. Most modification approaches aim at a special amino acid such as Lys and Cys, or $\mathrm{N}$-terminal amine [27]. There is no Lys in the TM3 (EGF4-6 (aa345-aa465)). Therefore, we chose $N$-terminal amine as the site-selective incorporation of azide group via

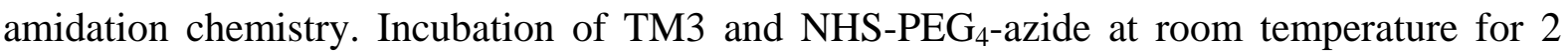
hours followed by removal of excess $\mathrm{NHS}_{-} \mathrm{PEG}_{4}$-azide by desalting column afforded the desired azido-TM3-N, which was confirmed by labeling with DBCO-Cy5 as well. As a result, after the fluorescent labeling, the azido-TM3-N showed a bright red band as detected by fluorescent scanner, while non-labeled one did not show any fluorescent image (Figure 4A). In addition, in order to compare the yields of two single-site azide incorporation methods, the fluorescent intensities of DBCO-Cy5 labeled azido-TM3-S and azido-TM3-N were compared by Image J. As a result, the fluorescent intensity ratio of azido-TM3-S to azido-TM3-N is 1:1, indicating these two methods afford similar reaction yields.

\section{Figure 4}

Recently, tyrosine becomes an attractive target for protein modification because of its relatively infrequent occurrence and ability to be modified without altering protein charge [28,29]. One tyrosine-selective modification is the three-component Mannich type reaction, which uses aniline derivatives and aldehyde for highly selective tyrosine residue modification at the ortho position on phenol group, particularly it has been demonstrated that the method condition affects little if any of protein activity [28]. In our recent study, we demonstrated that the three-component Mannich type reaction occurs in mild conditions and low millimolar concentration of reagents at $\mathrm{pH} 5.5-6.5$ and results in high yield [29]. TM3 contains three tyrosine residues and its multiple azide modification was investigated in this study. Briefly, 
4-azidoaniline, formaldehyde, and TM3 were added to 0.1 M PBS buffer ( $\mathrm{pH}$ 6.5) and incubated for 48 hours at room temperature. After desalting column, the resultant conjugate (azido-TM3-T) was obtained and was fluorescent labeled with DBCO-Cy5 through CFCC to verify the incorporation of azide as above. As a result, the azido-TM3-T showed a bright red band as detected by fluorescent scanner, while non-labeled TM3 did not show any fluorescent image (Figure 4B). Also, more than one tyrosine residue was modified on TM3 (S3). These results indicated that tyrosine-targeted methods can be used to incorporate azido functionality into the TM3 on multi-sites.

\subsection{Protein C activation activity of azido-TM456.}

After successfully prepared four kinds of azido- $\mathrm{TM}_{456} \mathrm{~S}$ via multiple approaches above, we investigated the effect of the azide group on the activity of $\mathrm{TM}_{456}$. TM is a potent activator of protein C. Subsequently, the activated form of protein C (APC), an anticoagulant protease, selectively inactivates coagulation factors $\mathrm{Va}$ and VIIIa. In this study, protein $\mathrm{C}$ activation activities of these azido- $\mathrm{TM}_{456}$ derivatives were measured and compared with the unmodified TM3. The absorptions of cleaved chromogenic substrates by APC were measured at $405 \mathrm{~nm}$. As a result, all the azido-TM456 derivatives showed high APC generation activities (Figure 5). Compared with unmodified TM3, azide modified $\mathrm{TM}_{456}$ by site-selective methods (azido-TM2, azido-TM3-S and azido-TM3-N) gave comparable activities, indicating that neither terminus nor near terminus modification of $\mathrm{TM}_{456}$ by a single azide molecule affect the $\mathrm{TM}_{456}$ activity. However, azido-TM3-T showed a slightly decreased activity, which may be due to the multi-site modifications on tyrosine residues of $\mathrm{TM}_{456}$ that affect either thrombin or PC interaction related to the protein $\mathrm{C}$ activation.

\section{Figure 5}




\subsection{Immobilization of azido-TM456 on DBCO-coated glass}

TM is mainly expressed on the surface of endothelial cell as an essential protein for the regulation of coagulation. Thus, immobilizing TM on biomaterial surface has been explored for developing antithrombotic biomaterials [30-35]. Compared with full TM, $\mathrm{TM}_{456}$ is more suitable to prepare anticoagulant surface due to its smaller molecular weight and purer anticoagulant activity. In this study, we examined the four different azido- $\mathrm{TM}_{456}$ derivatives obtained above for immobilization onto DBCO-coated glass so as to demonstrate their potential for biomaterial surface functionalization applications. For azido-TM2, we prelabeled it with FITC, and then incubated with the DBCO-coated glass slide. After washing, the slide was scanned by fluorescent scanner and green spots were observed clearly (Figure 6C), while the slide with TM2 (Figure 6B) that did not contain the azide group to react with DBCO showed nothing. Similarly, azide modified TM3s (azido-TM3-S, azido-TM3-N and azido-TM3-T) were immobilized on the DBCO-coated glass slide via CFCC. The immobilized TM3s were later detected by anti-TM antibody and FITC-labeled anti-mouse secondary antibody. Green spots shown indicated that all three azide modified TM3s were successfully immobilized on the glass via CFCC reaction (Figure 6E, 6F, 6G), and no significant differences were observed among them. Further, protein $\mathrm{C}$ activation activities of these immobilized azido- $\mathrm{TM}_{456}$ derivatives were measured and compared with the unmodified $\mathrm{TM}_{456} \mathrm{~S}$ (Figure 7). Consistently, $\mathrm{TM}_{456}$ with a single azide group (azido-TM2, azido-TM3-S and azido-TM3-N) gave comparable activities, indicating that site-specific immobilization via either terminus or near terminus of $\mathrm{TM}_{456}$ did not affect the $\mathrm{TM}_{456}$ activity. However, azido-TM3-T showed a slightly decreased activity, which may be due to the random immobilization through multiple azide group conjugated to tyrosine residues of $\mathrm{TM}_{456}$ that affect either thrombin or $\mathrm{PC}$ interaction related to the protein $\mathrm{C}$ activation. 


\section{Figure 6}

\section{Figure 7}

\section{Discussion}

Azide is the key group in the bio-orthogonal reaction, and is often the first choice biomolecule modification via CFCC. Therefore, there is a growing demand of methods to introduce this small azide functionality to biomoleucles easily and efficiently. Even more, to control the specific site of which amino acid is modified would benefit more, since this will maximally avoid reducing the activity of modified protein. In this study, we incorporated a single azide group to $\mathrm{TM}_{456}$ by targeting one specific amino acid at either $N$-terminus or $C$ terminus. The most predominant way is to use methionine auxotrophic E. coli strain to express azido-protein by replacing the natural amino acid methionine with unnatural amino acid azidohomoalanine. However, the protein expression requires prokaryotic expression system, whereas a human protein such as TM containing multiple disulfide bonds often suffers a lower yield recombinant expression. As we found, the direct expression yield of azido- $\mathrm{TM}_{456}$ is quite lower than native one (Table 1). Therefore, in order to increase the total amount of azido- $\mathrm{TM}_{456}$ production, we tried to use post-expression modification to introduce azide group to $\mathrm{TM}_{456}$ expressed with native amino acids via enzymatic and chemical reaction.

The enzymatic ligation we used in the study is sortase-mediated ligation in which the poly-(glycine) containing molecule is added to protein with LPEXG motif. SrtA kinetic study shows that SML requires high concentration of oligoglycine containing substrate to reach satisfying ligation efficiency. Besides, when the substrate is large or poor soluble in water, 
the acyl-enzyme intermediate (LPXTG-SrtA) exists, which is hardly resolved by the oligoglycine but finally hydrolyzed by water $[19,36]$. This is the major problem of SML when used for larger molecules conjugation. Therefore, the substrate containing the desired functionality needs to be designed properly in order to minimize the formation of the intermediate. Pentelute et al. utilized SML to generate protein thioester by peptide1-LPETG and $\mathrm{G}_{\mathrm{n}}$-thioester, followed by native chemical ligation (NCL) to link to the peptide with $\mathrm{N}$ terminal Cys [37]. This sequential ligation takes the advantage of site-specific SML using soluble and small oligoglycine substrate. Similarly, we used SML to introduce small water soluble $\mathrm{PEG}_{3}$-azide group to $\mathrm{TM}_{456}$, allowing the further protein modification by large, poor water soluble molecules through CFCC. Another feature is that fusion $\mathrm{TM}_{456}$ and SrtA with a His-tag at the $C$-terminus facilitates a easy cleanup of the unreacted TM3 and SrtA after the SML reaction. In the reaction mixture only the azide modified TM3 does not have His-tag, thus using nickel affinity column could remove the unreacted TM3 and SrtA easily so as to afford the pure azido-TM3 efficiently (Figure 3A). Therefore, this is another advantage of using SML to introduce azide group to TM3 comparing with the other modifications resulting in a mixture of native TM3 and azido-TM3. As a result, azido-TM3-S obtained in two steps with much higher yield comparing with the direct azido-TM3 expression using azidohomoalanine (Table 1), which will facilitate site-selective modification of TM3 in large amount for practical applications.

\section{Conclusions}

Herein, we report a multiple strategies for site-selectively introducing azide functionality into recombinant $\mathrm{TM}_{456}$, via direct recombinant expression with unnatural amino acid, chemical, and SML modification for its bio-orthogonal modification application. Overall, SML of recombinant protein affords the highest overall yield for incorporating azide functionality 
into recombinant $\mathrm{TM}_{456}$ at the $C$-terminus since the key protein expression step uses natural amino acids. As mentioned above, $\mathrm{TM}_{456}$ is a small protein containing 133 amino acid while has both thrombin and protein $\mathrm{C}$ binding domain. Therefore, in order to retain most of its bioactivity, the best choice is to modify the $N$ - or $C$ - terminal of $\mathrm{TM}_{456}$. This hypothesis was confirmed by our results that single modification maintained the protein's activity, while the multiple modifications retained less than $80 \%$ of the activity. In general, we have demonstrated a practical method for introducing azide functionality into recombinant protein for its site-selective modification application, all of which can be applied to proteins of different interests for both basic research and application. 


\section{Abbreviations:}

CFCC, Copper-free click chemistry; EGF, Epidermal growth factor; GG, Glycineglycine;

PC, Human protein C; PEG, Poly(ethylene) glycol; SML, Sortase-mediated ligation; TM, Thrombomodulin

\section{Acknowledgments}

This work was supported by grants from the American Heart Association (AHA) (14GRANT20290002, X.-L. Sun), NIH (1R01HL102604-04, X.-L. Sun), National Science Foundation (CHE-1126384, X.-L. Sun), Faculty Research Fund from the Center for Gene Regulation in Health and Disease (GRHD) (X.-L. Sun) at Cleveland State University supported by Ohio Department of Development (ODOD). 


\section{REFERENCES}

1. Kiick KL, Saxon E, Tirrell DA, Bertozzi CR. Incorporation of azides into recombinant proteins for chemoselective modification by the Staudinger ligation. Proc Natl Acad Sci USA. 2002;99:19-24.

2. Sun XL, Stabler CL, Cazalis CS, Chaikof EL. Carbohydrate and protein immobilization onto solid surfaces by sequential Diels-Alder and azide-alkyne cycloadditions. Bioconjug Chem. 2006;17:52-7.

3. Bostic HE, Smith MD, Poloukhtine AA, Popik VV, Best MD. Membrane labeling and immobilization via copper-free click chemistry. Chem Commun (Camb.) 2012;48:1431-3.

4. Prescher JA, Dube DH, Bertozzi CR. Chemical remodeling of cell surfaces in living animals. Nature. 2004;430:873-7.

5. Prescher JA, Bertozzi CR. Chemistry in living systems. Nat Chem Biol. 2005;1:1321.

6. Saxon E, Bertozzi CR. Cell surface engineering by a modified Staudinger reaction. Science. 2000;287:2007-10.

7. Wang Q, Chan TR, Hilgraf R, Fokin VV, Sharpless KB, Finn MG. Bioconjugation by copper(I)-catalyzed azide-alkyne $[3+2]$ cycloaddition. J Am Chem Soc. $2003 ; 125: 3192-3$.

8. Baskin JM, Prescher JA, Laughlin ST, Agard NJ, Chang PV, Miller IA, et al. Copperfree click chemistry for dynamic in vivo imaging. Proc Natl Acad Sci USA. 2007;104:16793-7.

9. Martin FA, Murphy RP, Cummins PM. Thrombomodulin and the vascular endothelium: insights into functional, regulatory, and therapeutic aspects. Am J Physiol Heart Circ Physiol. 2013;304:H1585-97. 
10. Weiler H, Isermann BH. Thrombomodulin. J Thromb Haemost. 2003;1:1515-24.

11. Wang, L, Jiang R, Sun XL. Recombinant thrombomodulin of different domains for pharmaceutical, biomedical, and cell transplantation applications. Med Res Rev. 2014;34:479-502.

12. Okamoto T, Tanigami H, Suzuki K, Shimaoka M. Thrombomodulin: a bifunctional modulator of inflammation and coagulation in sepsis. Crit Care Res Pract. 2012;2012:614545.

13. Cazalis CS, Haller CA, Sease-Cargo L, Chaikof EL. C-terminal site-specific PEGylation of a truncated thrombomodulin mutant with retention of full bioactivity. Bioconjug Chem. 2004;15:1005-9.

14. Stabler CL, Sun XL, Cui W, Wilson JT, Haller CA, Chaikof EL. Surface reengineering of pancreatic islets with recombinant azido-thrombomodulin. Bioconjug Chem. 2007;18:1713-5.

15. Wilson JT, Haller CA, Qu Z, Cui W, Urlam MK, Chaikof EL. Biomolecular surface engineering of pancreatic islets with thrombomodulin. Acta Biomater. 2010;6:1895903.

16. Zhang H, Weingart J, Jiang R, Peng J, Wu Q, Sun XL. Bio-inspired liposomal thrombomodulin conjugate through bio-orthogonal chemistry. Bioconjug Chem. 2013;24:550-9.

17. Nagashima M, Lundh E, Leonard JC, Morser J, Parkinson JF. Alanine-scanning mutagenesis of the epidermal growth factor-like domains of human thrombomodulin identifies critical residues for its cofactor activity. J Biol Chem. 1993;268:2888-92.

18. Han HS, Yang SL, Yeh HY, Lin JC, Wu HL, Shi GY. Studies of a novel human thrombomodulin immobilized substrate: surface characterization and anticoagulation activity evaluation. J Biomater Sci Polym Ed. 2001;12:1075-89. 
19. Jiang R, Wang L, Weingart J, Sun XL. Chemoenzymatic bio-orthogonal chemistry for site-specific double modification of recombinant thrombomodulin. ChemBioChem. $2014 ; 15: 42-6$.

20. Proft T. Sortase-mediated protein ligation: an emerging biotechnology tool for protein modification and immobilisation. Biotechnol Lett. 2010;32:1-10.

21. Wu Z, Guo Z. Sortase-mediated transpeptidation for site-specific modification of peptides, glycopeptides, and proteins. J Carbohydr Chem. 2012;31:48-66.

22. Sijbrandij T, Cukkemane N, Nazmi K, Veerman EC, Bikker FJ. Sortase A as a tool to functionalize surfaces. Bioconjug Chem. 2013;24:828-31.

23. Wu Z, Guo X, Wang Q, Swarts BM, Guo Z. Sortase A-catalyzed transpeptidation of glycosylphosphatidylinositol derivatives for chemoenzymatic synthesis of GPIanchored proteins. J Am Chem Soc. 2010;132:1567-71.

24. Tanaka T, Yamamoto T, Tsukiji S, Nagamune T. Site-specific protein modification on living cells catalyzed by Sortase. ChemBioChem. 2008;9:802-7.

25. Huang X, Aulabaugh A, Ding W, Kapoor B, Alksne L, Tabei K, et al. Kinetic mechanism of Staphylococcus aureus sortase SrtA. Biochemistry. 2003;42:11307-15.

26. Rabuka D. Chemoenzymatic methods for site-specific protein modification. Curr Opin Chem Biol. 2010;14:790-6.

27. Sletten EM, Bertozzi CR. Bioorthogonal chemistry: fishing for selectivity in a sea of functionality. Angew Chem Int Ed Engl. 2009;48:6974-98.

28. Joshi NS, Whitaker LR, Francis MB. A three-component Mannich-type reaction for selective tyrosine bioconjugation. J Am Chem Soc. 2004;126:15942-43.

29. Wang L, Gruzdys V, Pang N, Meng F, Sun XL. Primary arylamine-based tyrosinetargeted protein modification. RSC Adv. 2014;4:39446-52. 
30. Jiang R, Weingart J, Zhang H, Ma Y, Sun XL. End-point immobilization of recombinant thrombomodulin via sortase-mediated ligation. Bioconjug Chem. 2012;23:643-9.

31. Li JM, Singh MJ, Nelson PR, Hendricks GM, Itani M, Rohrer MJ, et al. Immobilization of human thrombomodulin to expanded polytetrafluoroethylene. $\mathbf{J}$ Surg Res. 2002;105:200-8.

32. Sperling C, Salchert K, Streller U, Werner C. Covalently immobilized thrombomodulin inhibits coagulation and complement activation of artificial surfaces in vitro. Biomaterials. 2004;25:5101-13.

33. Wu B, Gerlitz B, Grinnell BW, Meyerhoff ME. Polymeric coatings that mimic the endothelium: combining nitric oxide release with surface-bound active thrombomodulin and heparin. Biomaterials. 2007;28:4047-55.

34. Yeh HY, Lin JC. Bioactivity and platelet adhesion study of a human thrombomodulin immobilized nitinol surface. J Biomater Sci Polym Ed. 2009;20:807-19.

35. Qu Z, Krishnamurthy V, Haller CA, Dorr BM, Marzec UM, Hurst S, et al. Immobilization of actively thromboresistant assemblies on sterile blood-contacting surfaces. Adv Healthc Mater. 2014;3:30-5.

36. Ton-That H, Liu G, Mazmanian SK, Faull KF, Schneewind O. Purification and characterization of sortase, the transpeptidase that cleaves surface proteins of Staphylococcus aureus at the LPXTG motif. Proc Natl Acad Sci USA. 1999;96:12424-29.

37. Ling JJ, Policarpo RL, Rabideau AE, Liao X, Pentelute BL. Protein thioester synthesis enabled by sortase. J Am Chem Soc. 2012;134:10749-52. 


\section{Graphical Abstract}

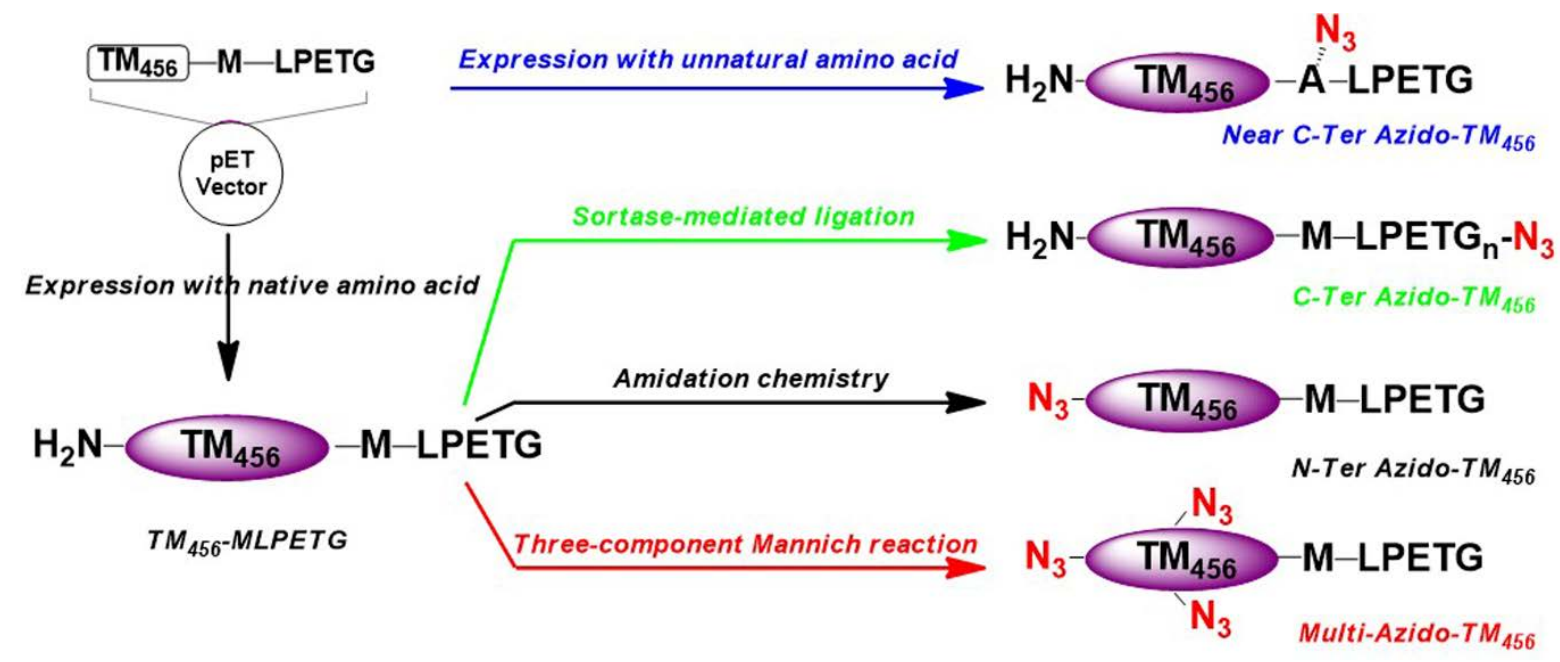

Four different strategies to introduce azide functionality into recombinant thrombomodulin (TM), site-specifically by recombinant, chemical, and enzymatic methods at either $\mathrm{N}$ terminus or C-terminus for its bio-orthogonal modification application. 
Table 1. Preparation yield of pure (azido-) $\mathrm{TM}_{456}$ per $1 \mathrm{~L}$ of cell culture expression

\begin{tabular}{ccc}
\hline $\begin{array}{c}\text { TM }_{456} \text { by } \\
\text { initial expression }\end{array}$ & $\begin{array}{c}\text { Azido-TM456 by post- } \\
\text { expression modification }\end{array}$ & $\begin{array}{c}\text { Final } \mathrm{TM}_{456} \text { amount } \\
\text { per liter expression (mg/L) }\end{array}$ \\
\hline TM2 & 6.2 \\
Azido-TM2 & 4.1 \\
TM3 & 13.5 \\
Azido-TM3 & 2.5 \\
& Azido-TM3-S & $8.1^{\text {a) }}$ \\
\hline
\end{tabular}

${ }^{a)}$ Final pure azido-TM3-S amount was calculated based on the yield of SML. 


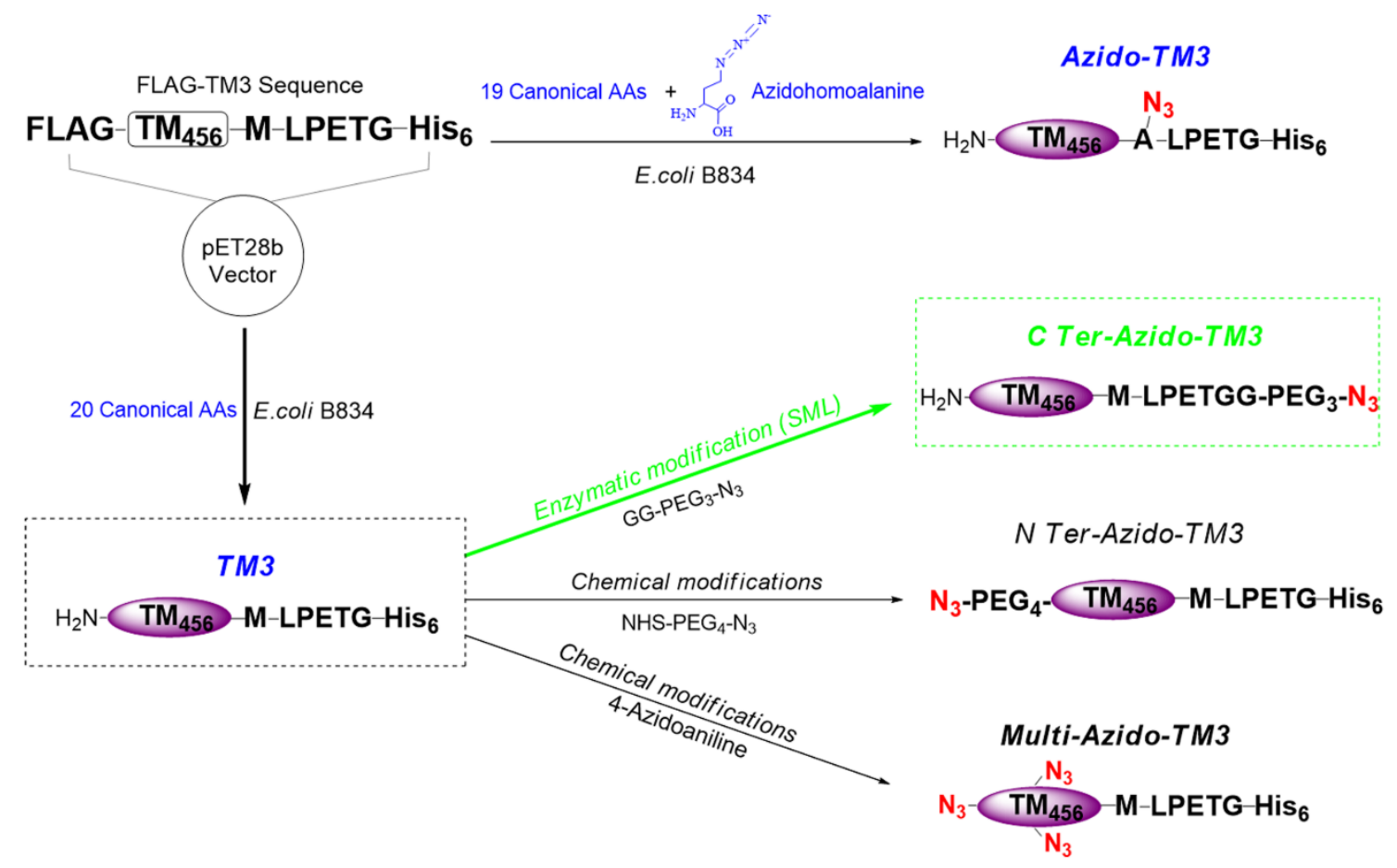

Figure 1. Multiple strategies for incorporating azide functionality into recombinant $\mathrm{TM}_{456}$ for its bio-orthogonal modification applications. (2 column) 
A



B

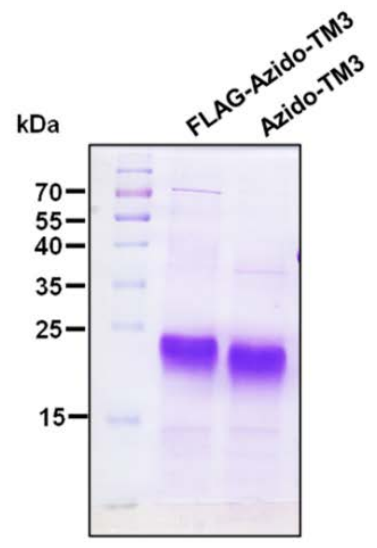

Figure 2. Expression of TM3 and azdio-TM3. (A): Lane 1, purified FLAG-TM3; Lane 2, purified TM3 after cleavage and removal of FLAG tag by enterokinase and nickel affinity chromatography. (B): Lane 1, purified azido-FLAG-TM3; Lane 2, purified azido-TM3 after cleavage and removal of FLAG tag by enterokinase and nickel affinity chromatography. ( 1.5 column ) 
A

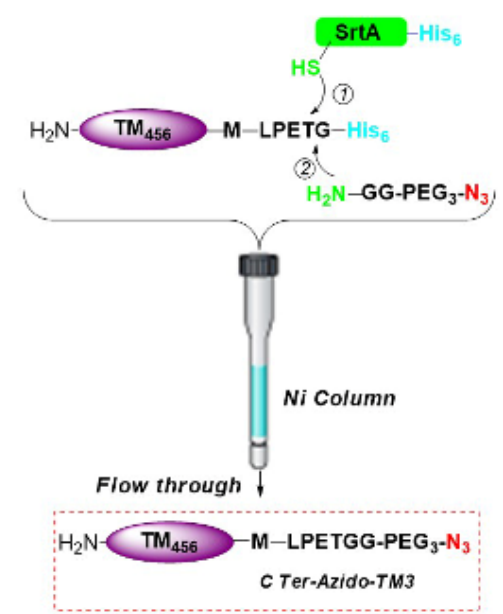

B

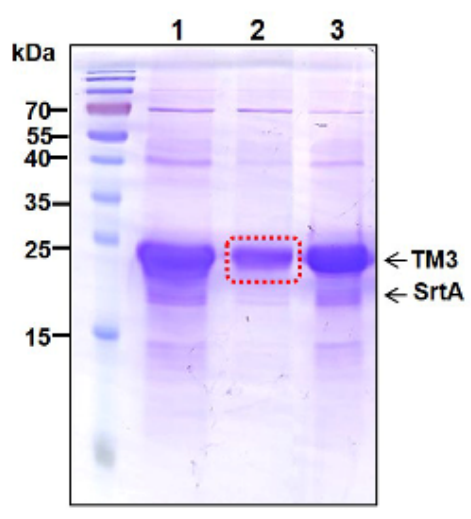

Figure 3. Preparation of azido-TM3-S by sortase-mediated ligation (SML). (A): Purification flowchart of SML reaction mixture. (B): SDS-PAGE of purification of azido-TM3-S. Lane 1, reaction mixture after desalting column; Lane 2, flow through (pure azido-TM3-S) from nickel affinity chromatography; Lane 3, eluate containing unreacted TM3 and SrtA from nickel affinity chromatography. (2 column) 
A

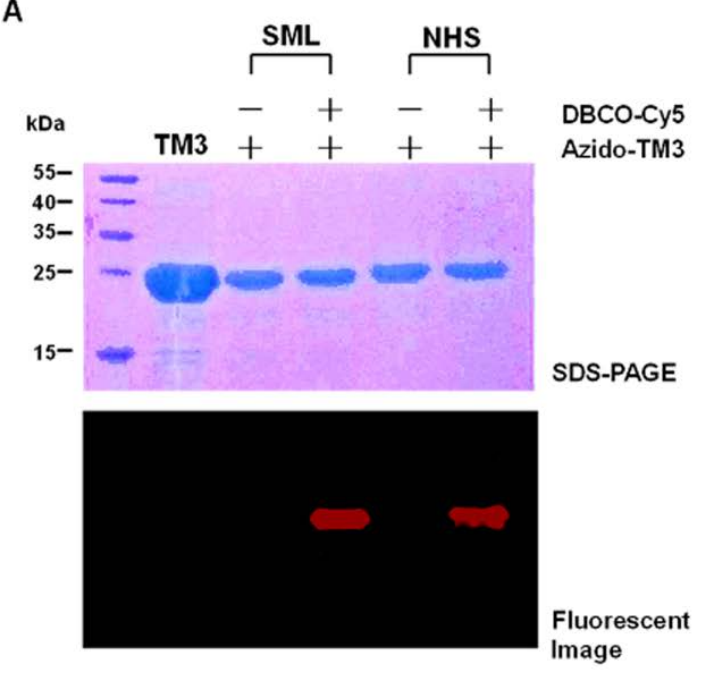

B



Figure 4. SDS-PAGE and fluorescent image of azide modified TM3s before and after reacted with DBCO-Cy5. (A): SDS-PAGE and fluorescent image of azido-TM3-S (prepared by SML) and azido-TM3-N (prepared by amidation chemistry) labeled by DBCO-Cy5 via CFCC. (B): SDS-PAGE and fluorescent image of azido-TM3-T (prepared by threecomponent Mannich reaction) labeled by DBCO-Cy5 via CFCC. (2 column) 


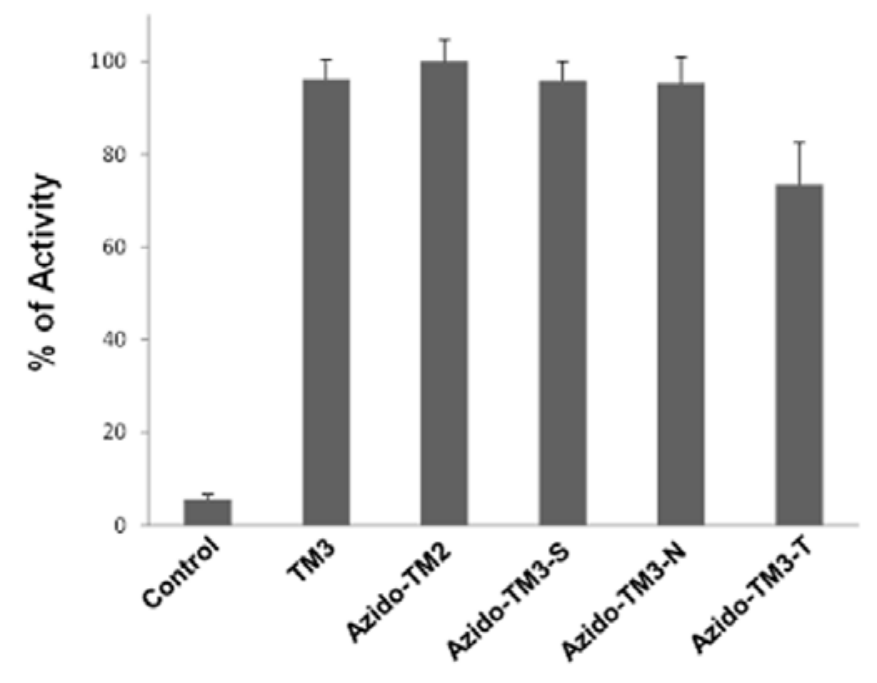

Figure 5. Protein $\mathrm{C}$ activation activities of TM3 and azido- $\mathrm{TM}_{456} \mathrm{~S}$. Data points represent the mean $\pm \mathrm{SD}$, for three independent measurements. Control group contained no $\mathrm{TM}_{456}$ at all. ( single column) 

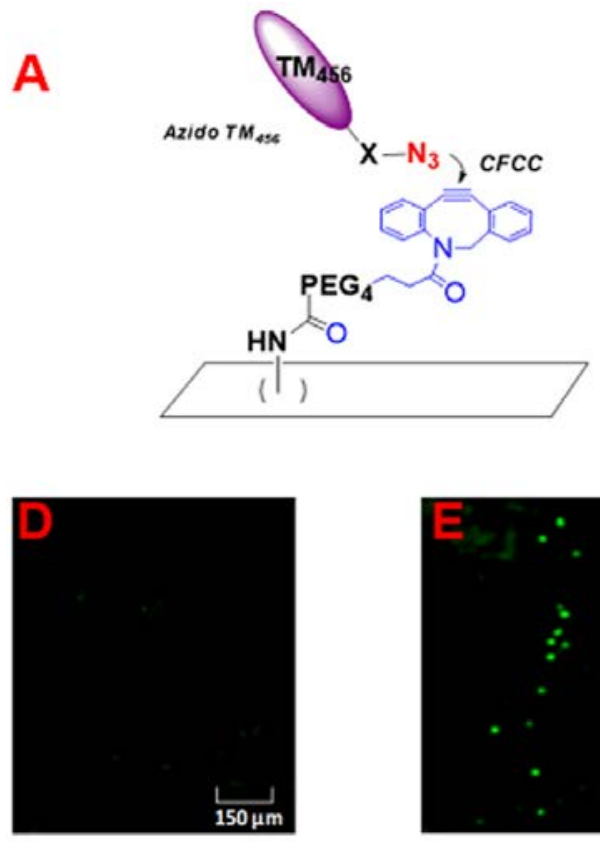

TM3



Azido-TM3-S



TM2



Azido-TM3-N

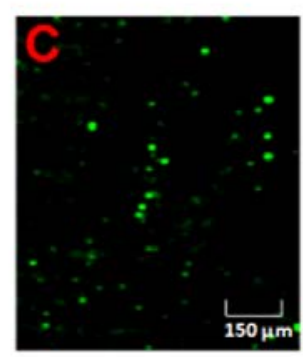

Azido-TM2

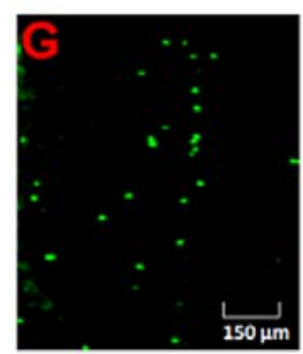

Azido-TM3-T

Figure 6. Immobilization of TM2, azido-TM2, TM3 and azido-TM3s onto DBCO-coated glass. (A): Schematic illustration of azido-TM3s immobilization via CFCC. (B): Control group using FITC labeled TM2 without azide for immobilization. (C): Immobilization of FITC labeled azido-TM2. (D): Control group using TM3 without azide for immobilization. (E): Immobilization of azido-TM3 obtained by SML. (F): Immobilization of azido-TM3 obtained by NHS ester reaction. (G): Immobilization of azido-TM3 obtained by threecomponent Mannich type reaction. For (D), (E), (F) and (G), the immobilized azido-TM3 derivatives were detected by mouse anti-TM antibody and FITC labeled anti-mouse secondary antibody. All images were obtained from Typhoon 9410 fluorescence scanner $(\mathrm{Ex} / \mathrm{Em}=480 / 520 \mathrm{~nm}) . \quad(2$ column $)$ 


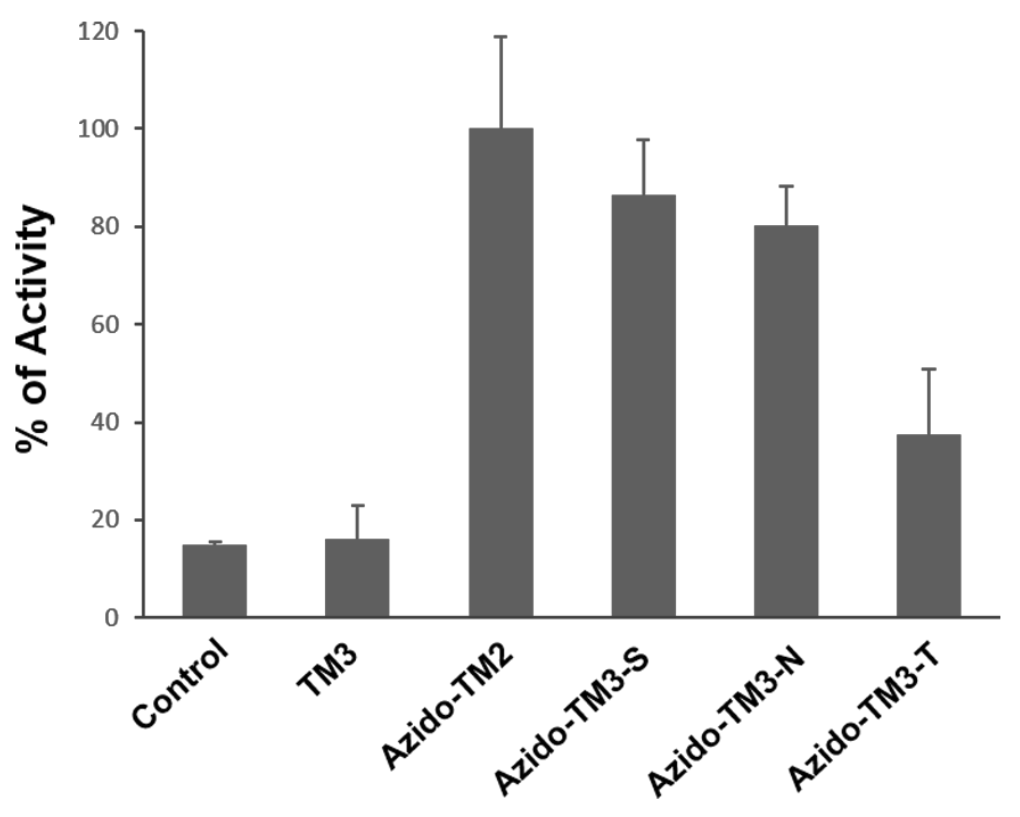

Figure 7. APC generation activities of immobilized azido-TM2 and azido-TM3s. Tris buffer and TM3 without azide were used to incubate with the DBCO-coated glass in group "Control" and "TM3" respectively. Data points represent the mean $\pm \mathrm{SD}$, for three independent measurements. (single column) 